\title{
Elaboración de productos de fibrocemento a partir de residuos de cuero.
}

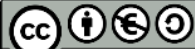

\section{Fiber cement products fabrication from leather residues.}

Calderón Segundo Hugo. ${ }^{1}$, Parada Rivera Mabel Mariela. ${ }^{2}$, Andrade Avalos Mónica Lilián ${ }^{3}$ $\&$ Borja Mayorga Danielita Fernanda ${ }^{4}$

Recibido: 27-05-2019 / Revisado: 25-06-2019 /Aceptado: 24-07-2019/ Publicado: 30-07-2019

\begin{abstract}
.
DOI: https://doi.org/10.33262/cienciadigital.v3i3.2.763

Substitution of chrysotile in fibro cement boards becomes relevant because it may be included as a dangerous product in the Ecuadorian legislation, due to its possible carcinogenic effects. The incorporation of leather residues (LR) in fibro cement boards were studied elaborating test probes containing 5\%, 6\%, 7\%, 10\% and $13.66 \%$ of leather residues. The quality properties considered were: modulus of rupture (MOR), bulk density (Bd) and absorption of water \% (Abs \%). The requirements were MOR > 4MPa (NTE INEN 2080:1996), Bd > 1, $37 \mathrm{~g} / \mathrm{ml}$ and \% Abs <28\% (Tubasec C.A. own standard). The $10 \%$ LR mix satisfied the máximum quality requirements (MOR=6,46 MPa, Bd=1,46 g/ml, \%Abs=27,88\%), including the Chi-square test of statistical significance $\left(\mathrm{CHI}^{2}=0,666\right)$. The $13,66 \% \mathrm{LR}$ mix had the best performance with respect to the modulus of rupture, but it did not satisfied the bulk density nor the absorption of water\% (MOR=6,53 $\mathrm{MPa}, \mathrm{Bd}=1,33 \mathrm{~g} / \mathrm{ml}, \% \mathrm{Abs}=34 \%)$.
\end{abstract}

\footnotetext{
${ }^{1}$ Escuela Superior Politécnica de Chimborazo, Facultad de Ciencias, Chimborazo, Ecuador hcalderon@espoch.edu.ec.

${ }^{2}$ Escuela Superior Politécnica de Chimborazo, Facultad de Ciencias, Chimborazo, Ecuador, mparada@espoch.edu.ec.

${ }^{3}$ Escuela Superior Politécnica de Chimborazo, Facultad de Ciencias, Chimborazo, Ecuador, moandrade@espoch.edu.ec.

${ }^{4}$ Escuela Superior Politécnica de Chimborazo, Facultad de Ciencias, Chimborazo, Ecuador, dborja@espoch.edu.ec.
} 
Keywords: Fiber cement, chrysotile, leather residues, construction material.

\section{Resumen}

El estudio de la sustitución del crisotilo, que se utiliza en láminas de fibro cemento, se hace pertinente ante la posibilidad de que dicho material sea incluido como producto peligroso dentro de la Normativa Ecuatoriana, por sus posibles efectos cancerígenos. Para estudiar la incorporación de residuos de cuero (RC) en las láminas se fabricaron probetas con 5\%, 6\%, 7\%, 10\% y 13,66\% de residuos de cuero. Las propiedades de calidad evaluadas fueron: módulo de rotura (MOR), densidad aparente (Da) y \% de absorción de agua (\%Abs). Los requisitos a cumplir fueron MOR >4MPa (NTE INEN 2080:1996), Da >1,37g/ml y \%Abs <28\% (Norma interna Tubasec C.A.). La mezcla con $10 \%$ de $\mathrm{RC}$ fue la que cumplió con la mayor cantidad de requisitos de calidad (MOR=6,46 $\mathrm{MPa}, \mathrm{Da}=1,46 \mathrm{~g} / \mathrm{ml}, \% \mathrm{Abs}=27,88 \%$ ), incluyendo la prueba que verifica la significancia estadística $\left(\mathrm{CHI}^{2}=0,666\right)$. La mezcla con 13,66\% de RC tuvo el mejor desempeño con respecto al módulo de rotura, pero estuvo fuera del rango referencial, tanto de la densidad aparente como del \% absorción de agua (MOR=6,53 $\mathrm{MPa}, \mathrm{Da}=1,33 \mathrm{~g} / \mathrm{ml}$, \% Abs=34 \%).

Palabras clave: Fibro cemento, crisotilo, residuos cuero, material construcción.

\section{Introducción.}

Los residuos sólidos de la industria del cuero aparecen como una alternativa al crisotilo en las láminas de fibrocemento, utilizadas en la construcción de viviendas como paredes interiores. El crisolito brinda a los productos de fibrocemento mayor resistencia, durabilidad y acabado. El crisotilo es un mineral que cristaliza en el sistema monoclínico o triclínico formando fibras. Su fórmula química es $\mathrm{Mg}_{3}\left(\mathrm{Si}_{2} \mathrm{O}_{5}\right)(\mathrm{OH})_{4}$ y se le conoce con el nombre de amianto o asbesto. La búsqueda de un material sustituto al crisotilo se debe a que se ha demostrado que es un material cancerígeno y existe la posibilidad que sea prohibido su uso en Ecuador.

En los años anteriores a 1988, los estudios realizados en personas que trabajaban en las minas o en fábricas, para relacionar el crisotilo como causante del mesotelioma, no había sido confirmado de manera categórica (Dunningan, 1988). Ahora bien, en estudios más recientes se estableció que los asbestos con crisotilo eran la causa de asbestosis y cáncer de pulmón (Smith y col., 1996) y también la causa de mesotelioma (Frank y col., 1998; Yano y col., 2009; Egilman y col., 2011). Otros estudios también confirmaron que en la fabricación de 
textiles, donde se usaba $100 \%$ crisotilo, el riesgo de desarrollar cáncer de pulmón era muy alto (Dement y col., 1994).

Entre los usos del crisotilo se pueden mencionar: aislante para tuberías y equipos, techos, lámina para paredes, pastillas de freno, aislante para cables eléctricos, pinturas antifuego, textiles, entre otros (Rowson, 1978; Virta, 2006). Los residuos de cuero han sido investigados para ser usados, en combinación con yeso, en la producción de láminas para cielos rasos (Faim y col., 2002). El uso de los residuos de cuero para ser incorporados en ladrillos de arcilla también ha sido estudiado (Aguiar y col., 2002). Aunque los ladrillos resultaron menos resistentes, su menor peso y mayor porosidad les permitiría ser usados como aislantes. Senthil y col., 2015 estudiaron la combinación de residuos de cuero con goma natural de latex para producir láminas, cuyo uso sería en suelas de zapatos y otros productos para recubiertas interiores del hogar.

Adicionalmente, debe mencionarse que los residuos de la industria del cuero pueden contener restos de cromo hexavalente el cual es peligroso para el medio ambiente. Por lo tanto, la incorporación de estos residuos en materiales de construcción o en fibras poliméricas, ayudaría a disminuir este problema de su eliminación (Sekaran y col., 2017; Lakraflia y col., 2012; El-Sabbagh y col., 2010).

En el afán de promover la mejora continua y la innovación, la búsqueda de nuevos materiales que puedan servir como sustitutos del crisotilo, se hace pertinente ante la posibilidad de que éste último sea incluido como material peligroso dentro de la Normativa Ecuatoriana.

En este orden de ideas, se plantea el estudio de la sustitución del crisotilo, que se utiliza en láminas de fibro cemento en la siguiente proporción en peso: $7 \%$ crisotilo, $76 \%$ cemento y $17 \%$ aditivos, por residuos de la industria del cuero.

\section{Procedimiento Experimental}

Materiales y métodos

Cemento Portland

Cemento Chimborazo Portland Puzolánico Tipo IP producido con Clinker, adiciones minerales y sulfato de calcio. Sus características técnicas se muestran en la Tabla 1.

Tabla 1. Características técnicas cemento Portland

\begin{tabular}{ll}
\hline Requisitos Químicos \\
\hline $\mathrm{MgO}(\%)$ & 1,99
\end{tabular}




\begin{tabular}{ll}
\hline $\mathrm{SO}_{3}(\%)$ & 1,75
\end{tabular}

Pérdida por ignición(\%) $\quad 2,14$

Requisitos Físicos

Peso específico $(\mathrm{g} / \mathrm{ml}) \quad 2,85$

Expansión en

autoclave $(\%)$

0,0013

Fraguado Vicat

Inicial(minuto)

160

Fraguado Vicat

Final(minuto)

Resistencia a

Compresión

3 días (MPa)

7 días $(\mathrm{MPa})$

28 días $(\mathrm{MPa})$

\section{Toba o roca volcánica}

Roca ígnea volcánica molida a un tamizado de malla N²00.

\section{Residuos o virutas de cuero}

Residuos sólidos de cuero provenientes de la empresa Curtiembre TUNGURAHUA. S.A., Riobamba, Ecuador. En la Figura 1 se muestra el material utilizado.

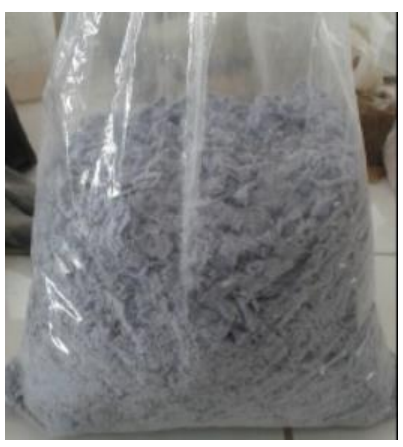

Figura 1. Virutas o residuos de cuero. 


\section{Aditivos}

Compuestos químicos confidenciales de la Empresa Tubasec C.A., Riobamba, Ecuador. La mezcla denominada estándar posee crisotilo $( \pm 7 \%)$ entre los aditivos.

\section{Elaboración de las probetas}

Para estudiar la posibilidad de sustituir el crisotilo por residuos de cuero en las láminas de fibrocemento, se elaboraron probetas con las diferentes proporciones de residuos o virutas de cuero y se compararon las semejanzas en calidad con las manufacturadas por la Empresa TUBASEC C.A. El procedimiento utilizado en la fabricación de las probetas se muestra en la Figura 2.

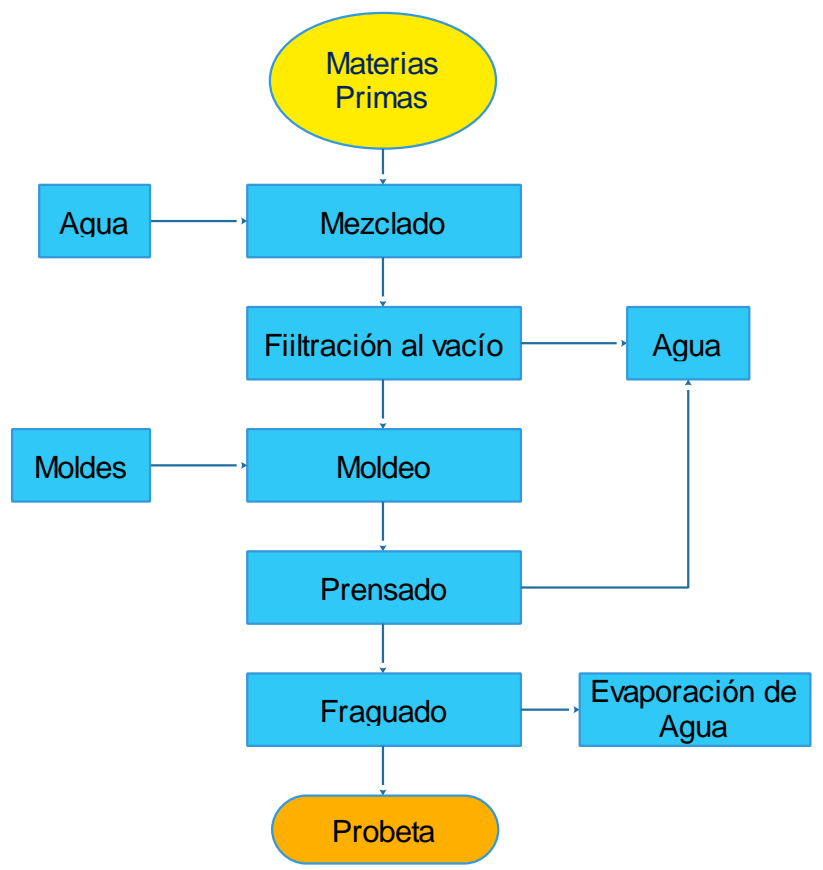

Figura 2. Procedimiento para la elaboración de las probetas.

El tamaño de las probetas fue fabricado de acuerdo a la norma NTE INEN 2080:1996. Las dimensiones de las mismas fue 250x250x20 mm. 


\section{Composición de las mezclas}

Las mezclas a ser estudiadas se muestran en la Tabla 2.

Tabla 2. Composición de las mezclas a ser estudiadas

\begin{tabular}{ccccc}
\hline & $\begin{array}{c}\% \\
\text { Cemento }\end{array}$ & $\begin{array}{c}\% \\
\text { Toba }\end{array}$ & $\begin{array}{c}\% \text { Res. } \\
\text { Cuero }\end{array}$ & $\begin{array}{c}\% \\
\text { Aditivos }\end{array}$ \\
\hline $\begin{array}{ccccc}\text { Estándar } \\
\text { Mezcla } \\
1\end{array}$ & 76,17 & 3,58 & - & 20,25 \\
Mezcla & 76,17 & 3,58 & 5 & 15,25 \\
2 & 76,17 & 3,58 & 6 & 14,25 \\
$\begin{array}{c}\text { Mezcla } \\
3\end{array}$ & 76,17 & 3,58 & 7 & 13,25 \\
$\begin{array}{c}\text { Mezcla } \\
4\end{array}$ & 76,17 & 2,09 & 10 & 11,74 \\
Mezcla & & & & \\
5 & 76,17 & 2,58 & 13,66 & 7,59 \\
\hline
\end{tabular}

\section{Propiedades físicas y mecánicas}

Una vez preparadas las probetas (siguiendo el procedimiento mostrado en la Figura 2) se procedió a seleccionar aquellas que no tuvieran grietas y que fueran las más uniformes en su espesor. Las propiedades de calidad evaluadas fueron: módulo de rotura, densidad aparente y absorción de agua (NTE INEN 2080, 1996; NTE INEN 2082, 1996). El módulo de rotura se midió utilizando una Máquina Universal de Ensayos (NESTOR) a una tasa de extensión de $5 \mathrm{~mm} / \mathrm{min}$. (Ver Figura 3). La densidad aparente de la probeta se calculó con el peso seco de la probeta (secado a $105^{\circ} \mathrm{C}$ por $24 \mathrm{hr}$ ) y su volumen medido por desplazamiento de líquido. La absorción de agua se determinó mediante la diferencia entre el peso seco y el peso saturado (después de $24 \mathrm{hr}$ sumergido en agua). 


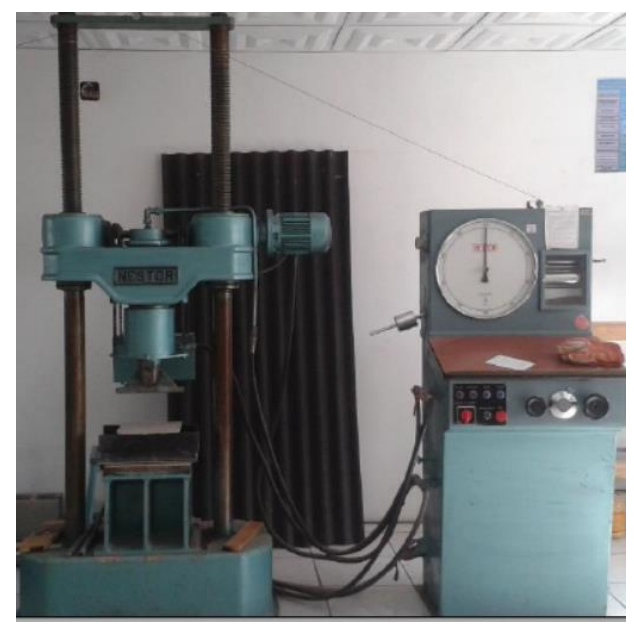

Figura 3. Máquina Universal de Ensayos

Se considera una relación factible de las variables de calidad, aquellas mezclas que mantengan las mismas tendencias en el tiempo de fraguado, con respecto a la mezcla estándar o patrón (con crisotilo).

\section{Normativa a cumplir}

Las probetas deben cumplir con las especificaciones que se muestran en la Tabla 3.

Tabla 3. Especificaciones a cumplir

$\begin{array}{cc}\text { NTE INEN } & \text { TUBASEC } \\ 2080 & \text { C.A. }\end{array}$

Módulo de Rotura

(MPa) Min.

4

10

Densidad Aparente

(g/ml) Min.

Absorción de Agua

(\%) Max. 


\section{Población de estudio}

Se seleccionaron 162 probetas, luego de descartar aquellas defectuosas, para las pruebas de calidad, 27 para cada mezcla y estándar (27x6). Se seleccionaron las 27 mejores para cada grupo; sin grietas, lo más planas posibles y espesor constante medido en varios puntos. Se escogieron nueve probetas para los distintos tiempos de fraguado, 7, 14 y 21 días respectivamente.

\section{Análisis Estadístico}

Se presentan los valores promedios y desviación estándar de las variables medidas. Se aplicó la prueba Chi Cuadrado ( $\mathrm{p}<0,5$ y 2 grados de libertad) a los valores de la MOR de las probetas estándar (7, 14 y 21 días) en conjunto con cada una de las composiciones de residuos de cuero, para la verificación de la independencia de dos variables y así verificar el cumplimiento de la normativa NTE INEN 2084:1996.

\section{Resultados y Discusión}

La primera prueba que se le realiza a las probetas es su resistencia a la flexión o módulo de rotura (MOR), el cual se calcula a través de la Ecuación 1:

$$
M O R=\frac{3 * P * L}{2 * A * e^{2}} * 100 \quad(E c n .1)
$$

$\mathrm{P}=$ Carga promedio $(\mathrm{N})$

$\mathrm{L}=$ Distancia entre apoyos (0,215 m NTE INEN 2080:1996)

$\mathrm{A}=$ Ancho de la probeta (0,25 m NTE INEN 2080:1996)

$\mathrm{e}=$ Espesor de la probeta $(\mathrm{m})$

El MOR se calcula para las probetas estándar y para cada una de las probetas con las mezclas consideradas $(5 \%, 6 \%, 7 \%, 10 \%$ y $13,66 \%$ de residuos de cuero, mezclas $1,2,3,4$ y 5 respectivamente). Las Figuras 4 y 5 muestran los valores del MOR en función de los días de fraguado considerados para los casos estudiados. Adicionalmente, se incluye el valor de la norma NTE INEN 2084:1996. 


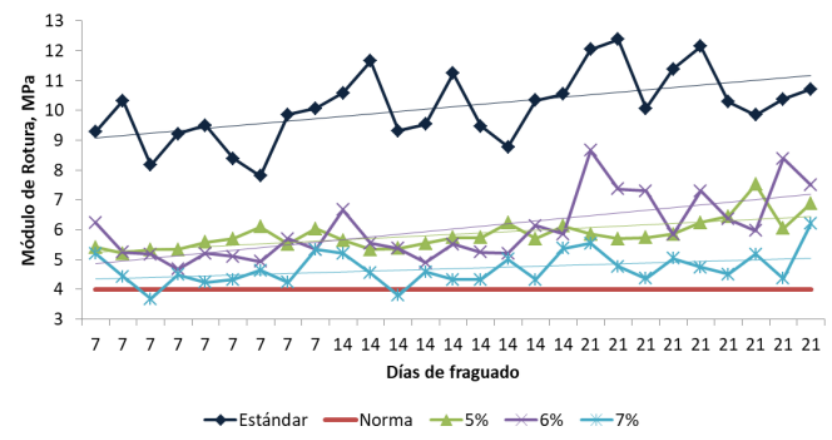

Figura 4. MOR de las probetas en función de los días de fraguado para las probetas estándar, Norma, 5\%, $6 \%$ y $7 \%$ de residuos de cuero.

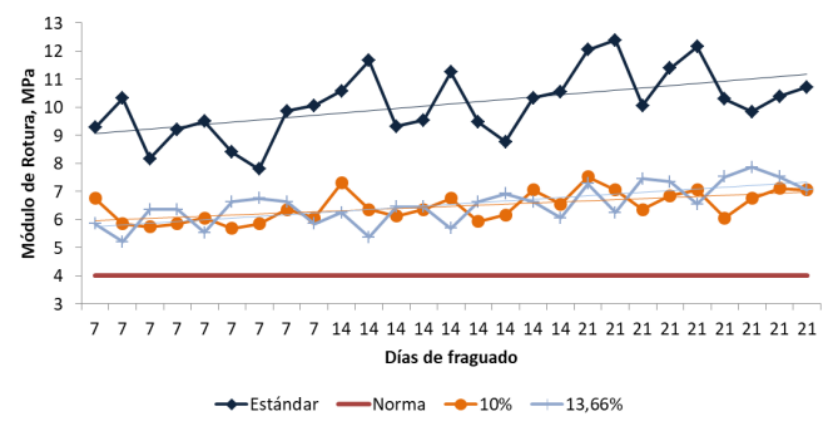

Figura 5. MOR de las probetas en función de los días de fraguado para las probetas estándar, Norma, $10 \%$ y $13,66 \%$ de residuos de cuero.

El primer aspecto a destacar al ver las Figuras 4 y 5 es la tendencia creciente del MOR a medida que aumenta el tiempo de fraguado, tanto para la probeta estándar como para todas las mezclas con cuero, lo cual es razonable debido a que mientras más seca la probeta mayor es la fuerza necesaria para romperla. Otro aspecto resaltante es que el MOR de la probeta patrón o estándar es mayor que todas las mezclas con residuos de cuero en todos los tiempos de fraguado, duplicándola en algunos casos. Finalmente, también se puede observar que todas las probetas de las mezclas cumplen con la Norma de $4 \mathrm{MPa}$, excepto 2 probetas de la mezcla de $7 \%$.

Los valores promedio y su desviación estándar para el MOR de todas las probetas se muestran en la Tabla 4. Como puede observarse en la Tabla 4, todos los valores promedio obtenidos cumplen con la norma NTE INEN 2084:1996, cuyo valor para láminas de fibrocemento Categoría 1, Tipo B es $4 \mathrm{MPa}$.

Tabla 4. Valores promedio y desviación estándar del MOR

\begin{tabular}{ccc}
\hline & MOR & Desviación \\
Probetas & Promedio, & \\
MPa & Estándar
\end{tabular}




\begin{tabular}{ccc}
\hline Estándar & 10,12 & 1,186 \\
Mezcla 1 & 5,85 & 0,510 \\
Mezcla 2 & 6,02 & 1,077 \\
Mezcla 3 & 4,70 & 0,558 \\
Mezcla 4 & 6,46 & 0,531 \\
Mezcla 5 & 6,53 & 0,692 \\
\hline
\end{tabular}

Adicionalmente, la prueba estadística $\mathrm{CHI}^{2}$ se aplicó a la serie de datos de todas las mezclas, tomando como relación la serie de datos de las probetas estándar, que cumple en forma excedida, con la norma NTE INEN 2084:1996. Las filas (3) de datos considerados fueron los tiempos de fraguado (7, 14 y 21 días) y las columnas (2) los MOR de las probetas estándar y los MOR de las probetas con residuos de cuero. Se consideró como hipótesis nula, $\mathrm{H}_{0}$, el cumplimiento con la norma NTE INEN 2084 y como hipótesis 1, $\mathrm{H}_{1}$, el no cumplimiento con la norma. El valor de $\mathrm{CHI}^{2}$ teórico para 2 grados de libertad y una probabilidad mayor de $50 \%$ es 1,3863 . $\mathrm{Si} \mathrm{CHI}^{2}$ calculado< $\mathrm{CHI}^{2}$ teórico, se rechaza $\mathrm{H}_{1} . \mathrm{Si} \mathrm{CHI}^{2}$ calculado $>\mathrm{CHI}^{2}$ teórico, se rechaza $\mathrm{H}_{0}$. En la Tabla 5 se enseña el análisis $\mathrm{CHI}^{2}$.

Tabla 5. Prueba $\mathrm{CHI}^{2}$ para los datos de los distintos contenidos de residuos de cuero

\begin{tabular}{|c|c|c|c|}
\hline & $\mathrm{CHI}^{2}$ & & $\mathrm{CHI}^{2}$ \\
\hline & Calculado & & Teórico \\
\hline \multicolumn{4}{|c|}{ Mezcla } \\
\hline 1 & 1,827 & $>$ & 1,3863 \\
\hline \multicolumn{4}{|c|}{ Mezcla } \\
\hline 2 & 1,761 & $>$ & 1,3863 \\
\hline \multicolumn{4}{|c|}{ Mezcla } \\
\hline 3 & 1,457 & $>$ & 1,3863 \\
\hline \multicolumn{4}{|c|}{ Mezcla } \\
\hline 4 & 0,666 & $<$ & 1,3863 \\
\hline \multicolumn{4}{|c|}{ Mezcla } \\
\hline 5 & 2,659 & $>$ & 1,3863 \\
\hline
\end{tabular}


La Tabla 5 muestra, que al usar la prueba estadística $\mathrm{CHI}^{2}$, la única mezcla que cumple con la norma NTE INEN 2084:1996 es la Mezcla 4, que corresponde a un contenido de residuos de cuero de $10 \%$.

La densidad aparente de las láminas de fibrocemento debe ser reportada por el fabricante. Para el caso que nos concierne, la empresa TUBASEC C.A. ha establecido un valor de referencia mínimo de $1,37 \mathrm{~g} / \mathrm{ml}$. Las Figuras 6 y 7 enseñan la densidad aparente de las probetas estándar, la norma y para cada una de las probetas con las mezclas consideradas $(5 \%, 6 \%, 7 \%, 10 \%$ y $13,66 \%$ de residuos de cuero, mezclas $1,2,3,4$ y 5 respectivamente).

La densidad aparente (Da) se calculó utilizando la ecuación 2, con la masa en gramos y el volumen desplazado en mililitros.

\section{Densidad Aparente $=\frac{\text { Masa seca }}{\text { Volumen desplazado de agua }}($ Ecn.2)}

En la Figura 6 se puede observar que la tendencia de la densidad de las probetas estándar es decreciente con el tiempo de fraguado. Las tendencias de las mezclas de 5\%, 6\% y $7 \%$ de cuero son crecientes, lo que es contrario al comportamiento del patrón. En relación al cumplimiento del valor referencial de $1,37 \mathrm{~g} / \mathrm{ml}$, todas las mezclas lo cumplen.

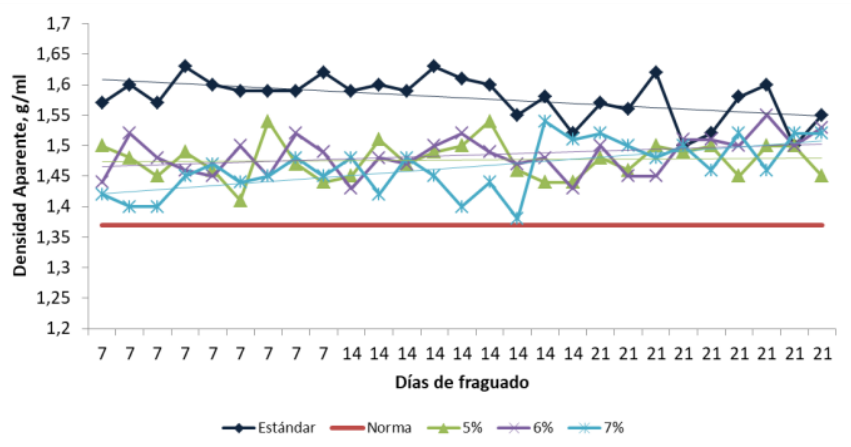

Figura 6. Densidad Aparente de las probetas en función de los días de fraguado para las probetas estándar, Norma, 5\%, 6\% y $7 \%$ de residuos de cuero.

En la Figura 7 se puede observar que las tendencias de las Da de las mezclas de $10 \%$ y $13,66 \%$ de cuero son decrecientes al igual que la tendencia de las probetas estándar, lo cual es el comportamiento deseado. Las probetas de $10 \%$ cumplen con la norma referencial de $1,37 \mathrm{~g} / \mathrm{ml}$ mientras las de $13,66 \%$, en su mayoría no cumplen. Se especula que la densidad del cuero estaría afectando los límites sugeridos al llegar a dicha proporción. 


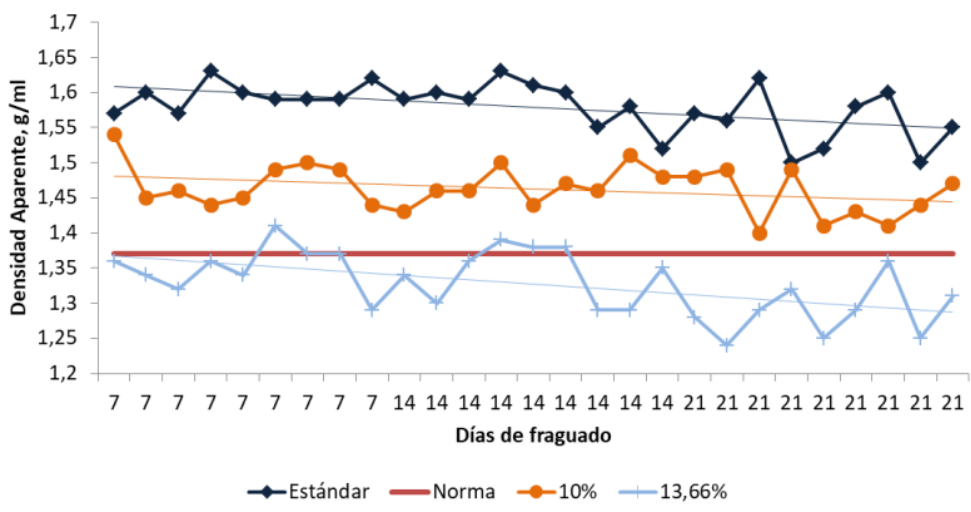

Figura 7. Densidad Aparente de las probetas en función de los días de fraguado para las probetas estándar, Norma, $10 \%$ y 13,66\% de residuos de cuero

En la Tabla 6 se observan los valores promedio y su desviación estándar de las densidades. Los datos muestran que en promedio la única mezcla que no cumple con el valor referencial es la de 13,66\% de cuero. Todas las mezclas resultan más livianas que la estándar y eso se explica por el contenido de cuero cuya densidad es menor.

Tabla 6. Valores promedio y desviación estándar de la densidad aparente de las probetas

\begin{tabular}{ccc}
\hline & $\begin{array}{c}\text { Densidad } \\
\text { Aparente }\end{array}$ & Desviación \\
\hline Probetas & $\begin{array}{c}\text { Promedio, } \\
\mathrm{g} / \mathrm{ml}\end{array}$ & Estándar \\
Estándar & 1,58 & 0,036 \\
Mezcla 1 & 1,48 & 0,031 \\
Mezcla 2 & 1,48 & 0,032 \\
Mezcla 3 & 1,46 & 0,044 \\
Mezcla 4 & 1,46 & 0,033 \\
Mezcla 5 & 1,33 & 0,046 \\
\hline
\end{tabular}


La cantidad de agua absorbida (\%Abs) por las probetas, luego de sumergida por 24 horas, se midió como otra variable de calidad de las láminas de fibrocemento. Esta propiedad está relacionada con la impermeabilidad y flexibilidad del material. La cantidad de agua se determinó como el porcentaje absorbido y se calculó mediante la ecuación 3.

$$
\% A b s=\frac{\text { Peso saturado }- \text { Peso seco }}{\text { Peso seco }} * 100(\text { Ecn. } 3)
$$

En la Figura 8 se puede observar que la tendencia del \% de absorción de agua de las probetas estándar es creciente con el tiempo de fraguado. Las tendencias de las mezclas de 5\%, 6\% y $7 \%$ de cuero son decrecientes, lo que es contrario al comportamiento del patrón, esto significa que estas proporciones de cuero se podrían estar consolidando de tal forma que su porosidad es menor con el paso del tiempo y por lo tanto absorben menos agua.

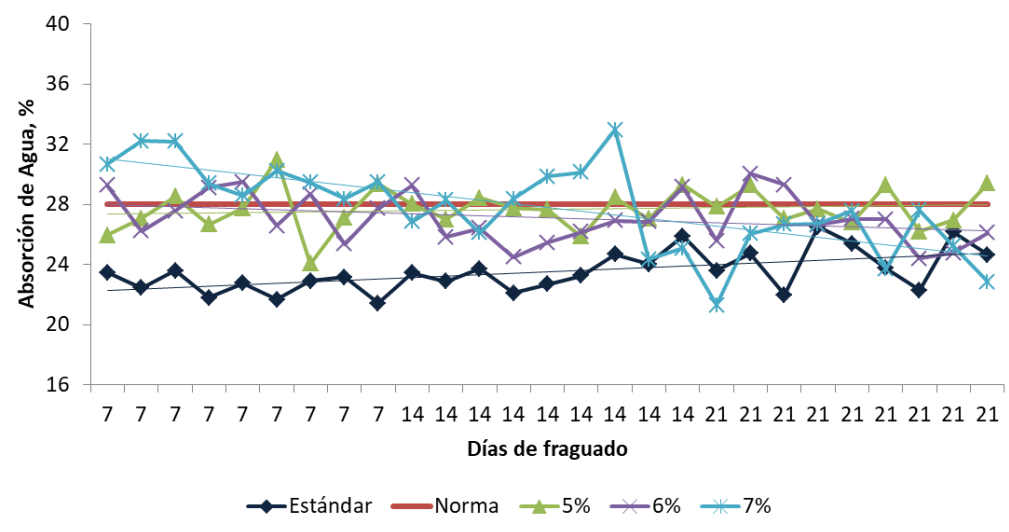

Figura 8. Absorción de agua de las probetas en función de los días de fraguado para las probetas estándar, Norma, $5 \%, 6 \%$ y $7 \%$ de residuos de cuero.

En la Figura 9 se puede observar que las tendencias del \% de absorción de agua de las mezclas de $10 \%$ y $13,66 \%$ de cuero son crecientes, igual al comportamiento del patrón, lo cual es la respuesta deseada. 


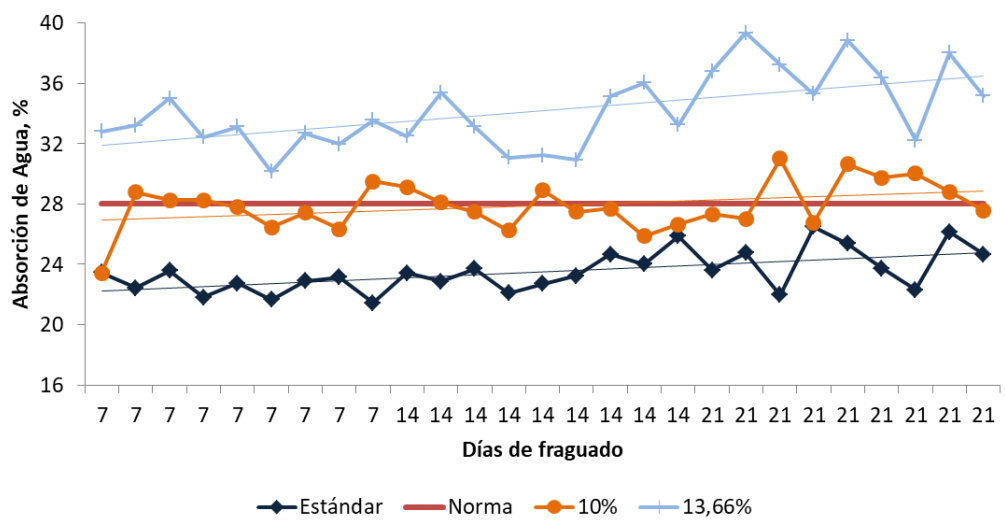

Figura 9. Absorción de agua de las probetas en función de los días de fraguado para las probetas estándar, Norma, $10 \%$ y $13,66 \%$ de residuos de cuero.

En la Tabla 7 se muestran los valores promedio y su desviación estándar del \% de absorción de agua. El valor referencial a cumplir es un \% de absorción máximo de $28 \%$. Los datos enseñan que en promedio la única mezcla que no cumple con el valor referencial es la de $13,66 \%$ de cuero (mezcla 5). Sin embargo, también puede notarse, que los valores promedio de las demás mezclas están muy cerca del valor referencial $(0,12 \%$ a $0,82 \%$ por debajo).

Tabla 7. Valores promedio y desviación estándar de la absorción de agua de las probetas

\begin{tabular}{ccc}
\hline & Absorción & Desviación \\
\hline Promedio, & \\
Probetas & $\%$ & Estándar \\
Estándar & 23,51 & 1,378 \\
Mezcla 1 & 27,68 & 1,422 \\
Mezcla 2 & 27,08 & 1,669 \\
Mezcla 3 & 27,78 & 2,896 \\
Mezcla 4 & 27,88 & 1,623 \\
Mezcla 5 & 34,18 & 2,488 \\
\hline
\end{tabular}




\section{Conclusiones}

Se pudo demostrar la factibilidad de utilizar residuos o virutas de la industria de cuero ecuatoriana como componente de las láminas de fibrocemento fabricadas en la empresa Tubasec C.A.

Aunque todas las mezclas cumplieron con el requisito del módulo de rotura (MOR>4 MPa, NTE INEN 2084:1996), las mezclas 5\%, 6\% y 7\% presentaron tendencias opuestas a la mezcla estándar (con crisotilo) tanto en la densidad aparente como en el \% absorción de agua, por lo cual quedaron descartadas por no cumplir con los criterios de calidad establecidos.

Las mezclas 4 (10\% cuero) y $5(13,66 \%$ de cuero) fueron las que cumplieron con los requisitos de calidad.

La mezcla 4, fue la que cumplió con la mayor cantidad de requisitos de calidad (MOR=6,46 $\mathrm{MPa}, \mathrm{Da}=1,46 \mathrm{~g} / \mathrm{ml}>1,37 \mathrm{~g} / \mathrm{ml}, \% \mathrm{Abs}=27,88 \%<28 \%$ ), incluyendo la prueba que verifica la significancia estadística $\left(\mathrm{CHI}^{2}=0,666<1,3863\right)$.

La mezcla 5 tuvo el mejor desempeño con respecto a la resistencia a la flexión o módulo de rotura, pero estuvo fuera del rango referencial, tanto de la densidad aparente como del $\%$ absorción de agua (MOR=6,53 MPa, $\mathrm{Da}=1,33 \mathrm{~g} / \mathrm{ml}<1,37 \mathrm{~g} / \mathrm{ml}, \% \mathrm{Abs}=34 \%>28 \%)$. Cabe mencionar que la norma NTE INEN 2080 solo exige reportar la densidad aparente y el \% de absorción de agua. Los valores mostrados son normas internas de la empresa Tubasec C.A.

\section{Referencias bibliográficas.}

Aguiar JB, Valente A., Pires MJ, Tavares T, 2002, Incorporation Feasibility of Leather Residues in Bricks, Key Engineering Materials, 206-213, pp. 2005-2008.

Dement JM, Brown DP, Okun A., 1994, Follow-Up Study of Chrysotile Asbestos Textile Workers: Cohort Mortality and Case-Control Analyses, American Journal of Industrial Medicine, 26, pp. 431-447.

Dunnigan J, 1988, Linking Chrysotile Asbestos with Mesothelioma, American Journal of Industrial Medicine, 14, pp. 205-209.

Egilman D, Menéndez LM, 2011, A Case of Occupational Peritoneal Mesothelioma From Exposure to Tremolite-Free Chrysotile in Quebec, Canada: A Black Swan Case, American Journal of Industrial Medicine, 54, pp.153-156.

El-Sabbagha SH, Mohamed OA, D’Amore A, Acierno D, Grassia L, 2010, Recycling of Chrome Tanned Leather Dust in Acrylonitrile Butadiene Rubber, AIP Conference Proceedings, pp. 249-251. 
Faim P, Ferreira J, 2002, Fabrication of New Building Materials from Leather Residues Agglomerated with Recycled Plaster, Key Engineering Materials, 230-232, pp. 428-431.

Francis W, Crayson J, 1941, Some fundamental properties of chrysotile asbestos in relation to electrical insulation, Journal of Chemical Technology \& Biotechnology, 60, 6, pp. 160166.

Frank AL, Dodson RF, Williams M.G., 1998, Carcinogenic Implications of the Lack of Tremolite in UICC Reference Chrysotile, American Journal of Industrial Medicine, 34, pp. 314-317.

Lakraflia H, Tahirib S, Albizanea A, El Otmani, ME, 2012, Effect of wet blue chrome shaving and buffing dust of leather industry on the thermal conductivity of cement and plaster based materials, Construction and Building Materials, 30, pp. 590-596.

Martin CJ, Phillips VA, 1977, The texture of chrysotile asbestos in relationship to mechanical properties, Materials Science and Engineering, 30, 1, pp. 81-87.

NTE INEN 2079, 1996, LÁMINAS PLANAS DE FIBRO-CEMENTO. DETERMINACIÓN DE LAS DIMENSIONES. Norma Técnica Ecuatoriana, Primera edición. Disponible en: http://apps.normalizacion.gob.ec/descarga/ (2018, 1 de Octubre).

NTE INEN 2080, 1996, LÁMINAS PLANAS DE FIBRO-CEMENTO. DETERMINACIÓN DEL MÓDULO DE ROTURA. Norma Técnica Ecuatoriana, Primera edición. Disponible en: http://apps.normalizacion.gob.ec/descarga/ (2018, 1 de Octubre).

NTE INEN 2082, 1996. LÁMINAS PLANAS DE FIBRO-CEMENTO. DETERMINACIÓN DE LA DENSIDAD. Norma Técnica Ecuatoriana, Primera edición. Disponible en: http://apps.normalizacion.gob.ec/descarga/ (2018, 1 de Octubre).

NTE INEN 2084, 1996. LÁMINAS PLANAS DE FIBROCEMENTO. REQUISITOS. Norma Técnica Ecuatoriana, Primera edición. Disponible en: http://apps.normalizacion.gob.ec/descarga/ (2018, 1 de Octubre).

Rowson DM, 1978, The chrysotile content of the wear debris of brake linings, Wear, 47, 2, pp. 315-321.

Senthil R, Hemalatha T, Manikandan R, Das BN, Sastry TP, 2015, Leather boards from buffing dust: a novel perspective, Clean Techn Environ Policy, 17, pp. 571-576.

Sivaprakash K, Maharaja P, Pavithra S, Boopathy R, Sekaran G, 2017, Preparation of light weight constructional materials from chrome containing buffing dust solid waste generated in leather industry, Journal of Material, Cycles and Waste Management, 19, pp. 928-938. 
Smith AH, Wright CC, 1996, Chrysotile Asbestos is the Main Cause of Pleural Mesothelioma, American Journal of Industrial Medicine, 30, pp. 252-266.

Virta RL, 2006, Worldwide asbestos supply and consumption trends from 1900 through 2003. Circular 1298. Reston (VA): United States Department of the Interior, United States Geological Survey.

Yano E, Wang Z, Wang X, Wang M, Takata A, Kohyama N, Suzuki Y, 2009, Mesothelioma in a Worker Who Spun Chrysotile Asbestos at Home During Childhood, American Journal of Industrial Medicine, 52, pp. 282-287. 


\section{PARA CITAR EL ARTÍCULO INDEXADO.}

Calderón, S. H., Parada Rivera, M., Andrade Lilián, M., \& Borja Mayorga, D. (2019). Elaboración de productos de fibrocemento a partir de residuos de cuero. Ciencia Digital, 3(3.2), 314-331. https://doi.org/10.33262/cienciadigital.v3i3.2.763

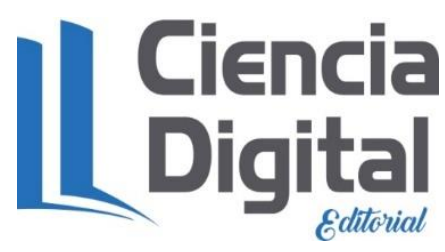

El artículo que se publica es de exclusiva responsabilidad de los autores y no necesariamente reflejan el pensamiento de la Revista Ciencia Digital.

El artículo queda en propiedad de la revista y, por tanto, su publicación parcial y/o total en otro medio tiene que ser autorizado por el director de la Revista Ciencia Digital.
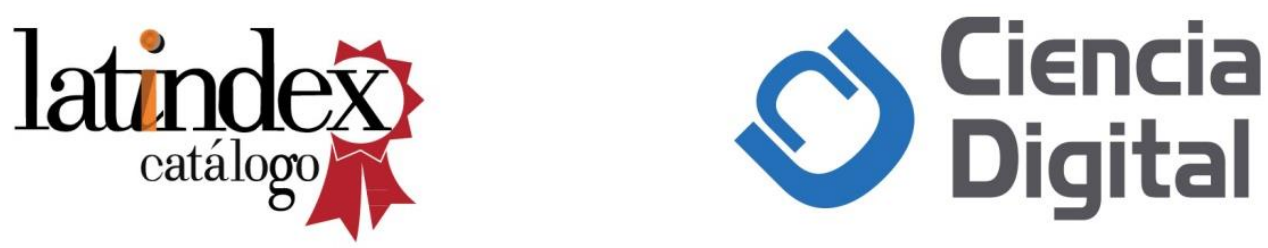